\title{
Analysis of Postoperative Pain at the Anterior Iliac Crest Harvest Site: A Prospective Study of the Intraoperative Local Administration of Ropivacaine
}

\author{
Juliane Zenner ${ }^{1}$, Wolfgang Hitzl ${ }^{2}$, Michael Mayer ${ }^{1}$, Heiko Koller ${ }^{1}$ \\ ${ }^{1}$ German Scoliosis Center Bad Wildungen, Werner-Wicker-Klinik, Bad Wildungen, Germany \\ ${ }^{2}$ Biostatistics, Research Office, Paracelsus Medical University Salzburg, Salzburg, Austria
}

Study Design: This was a prospective randomized comparative study.

Purpose: The aim of this study was to objectify donor site-related pain following anterior iliac crest graft harvesting, in patients who have undergone multilevel anterior cervical discectomy and fusion with plating (ACDFP); and to assess the effect of an intraoperative local single injection of ropivacaine on postoperative pain.

Overview of Literature: Multilevel ACDFP can be associated with a high non-union rate. Autogenous iliac bone has been used to increase union rates, although a high incidence of donor site-related pain has been reported.

Methods: Forty consecutive patients who required 3-level or 4-level ACDFP were prospectively assessed for donor site-related pain. Pain levels were assessed daily for five days postoperative using the visual analog scale (VAS). Patients were randomly assigned to group A or B. In group A patients, $7-10 \mathrm{~mL}$ of ropivacaine $(0.2 \%)$ was injected into the iliac crest after iliac crest graft harvesting. Morphine usage via patient controlled analgesia was calculated. At six months postoperative, patient complaints at the harvest site were documented.

Results: Patients were randomly assigned to group A or B. In group A, ropivacaine was locally administered at the site of the iliac crest graft harvest after fascia closure. In group $B$, no additional treatments were administered. The average patient age in group $A$ was $56 \pm 7.6$ years, whereas the average age of patients in group $B$ was $52.6 \pm 10.4$ years. Group $A$ had an average of $0.6 \pm 0.7$ previous surgeries per patient, whereas group $B$ had an average of $0.8 \pm 1.0$ previous surgeries per patient. The average number of levels fused in group $A$ was $3.6 \pm 0.7$, whereas the average number of levels fused in group $B$ was $3.7 \pm 0.9$ (all $p>0.05$ ). In group $A$, the mean ropivacaine volume administered was $8.4 \pm 1.5 \mathrm{~mL}$. No patient complaints regarding chronic pain, were reported six months postoperatively. No complications were encountered from the harvest site, and all patients underwent successful 3-level and 4-level ACDFP. Statistical analysis showed significant differences for VAS on postoperative day $1(p=0.004)$ and day $2(p=0.005)$.

Conclusions: VAS assessment showed overall moderate perioperative morbidity in terms of donor site-related pain, which was reduced by administering ropivacaine.

Keywords: Spine; Bone grafting; Complications; Local anesthetics; Pain assessment

\section{Introduction}

In anterior multilevel cervical spine surgery, autogenous bone from the anterior iliac crest can be used to foster union. Pseudarthrosis rates in patients undergoing multilevel surgery have been reported in as many as $30 \%$ of pa-

Received Apr 29, 2014; Revised May 19, 2014; Accepted May 20, 2014

Corresponding author: Juliane Zenner

German Scoliosis Center, Werner-Wicker-Klinik, Im Kreuzfeld 4, 34537 Bad Wildungen, Germany

Tel: +49-1511-6723680, Fax: +49-5621-80320, E-mail: juliane.zenner@gmx.de 
tients $[1,2]$. Autogenous bone grafting has been the method of choice to achieve fusion for many years. However, reports of complications have led to a widespread search for alternatives. While allografts have the disadvantage of higher costs and lower fusion rates, autogenous bone grafts are easily available at low cost and harbor osteoinductive, osteoconductive and nonimmunogenic properties while avoiding the potential for interpatient disease transmission [3]. The use of bone morphogenetic proteins (BMPs) in cervical spine fusion was correlated with an increased rate of dysphagia, hospital costs and overall complications, including life-threatening airway complications [4-7]. Accordingly, the use of BMPs has become doubtful, and the best way in which to foster union has become an area of increasing interest.

Although iliac crest graft (ICG) harvesting is thought to confer high perioperative morbidity with significant donor site-related pain hampering the postoperative recovery of patients, we have found little evidence to support this presumption. The intraoperative administration of local anesthetics has been described; however, the effects of local anesthetics on multilevel cervical spine surgery have not yet been defined. We wished to further clarify these allegations in patients with multilevel plated anterior cervical discectomy and fusion (ACDFP) and to identify a way to ameliorate postoperative pain. The objective of the current study was therefore, to prospectively compare the approach-related morbidity in terms of postoperative pain levels with and without the intraoperative local application of ropivacaine to the ICG harvest site.

\section{Materials and Methods}

This study was a prospective assessment of 40 consecutive patients undergoing 3-level and 4-level ACDFP within a one-year period. Patients were randomized in alternating order to group A or B. Inclusion criteria were 3- or 4-level ACDFP with anterior iliac crest bone graft harvesting, complete VAS scores for the first five days postoperative, and available radiographs at 6 months follow-up. Patients with a history of lumbar surgery, total hip or knee arthroplasty, previous abdominal surgery on the side of the bone graft harvest or previous iliac crest bone graft harvesting were excluded. Surgery was performed by two surgeons. In group A, the average patient age at the time of surgery was 56 years (range, 43-68 years), whereas the average patient age of group B was 53 years (range, 37-70 years). The male-to-female ratio was 8:12 in group A and 7:13 in group B. Indications for surgery were axial neck pain, myeloradiculopathy and failed surgery elsewhere. In group A, 11 patients showed cervical spondylosis and instability with or without myeloradiculopathy, five patients suffered from pseudarthrosis, and two patients presented with a failed cervical disc replacement. In group B, 14 patients suffered from cervical spondylosis and instability, five patients suffered from pseudarthrosis, one patient presented with a failed cervical disc replacement and one patient presented with a malpositioned cage. Group A had an average of $0.6 \pm 0.7$ previous surgeries per patient (range, $0.0-2.0$ ), whereas group B had an average of $0.8 \pm 1.0$ per patient (range, $0.0-3.0$ ). In each group, seven patients underwent implant removal. One corpectomy of the $\mathrm{C} 4$ vertebra was performed in group $\mathrm{A}$, and one corpectomy of the $\mathrm{C} 5$ vertebra was performed in group B. All patients were treated with multilevel ACDFP. In group $\mathrm{A}$, two patients had an additional staged posterior augmentation of the anterior construct. The average number of levels fused in group A was 3.6 \pm 0.7 (range, $2.0-5.0$ ), whereas the average number of levels fused in group B was 3.7 \pm 0.9 (range, 2.0-6.0). Statistical analysis did not reveal significant differences regarding baseline characteristics in groups A and B $(p<0.05)$.

In all cases, anterior ICG harvesting was performed by established institutional standards (Fig. 1). Surgery was performed by two of the authors, using identical techniques. Patients were randomly assigned to group A or group B. After completion of bone grafting and fascia closure, a local anesthetic was administered to the harvest site in the 20 consecutive patients in group A. In the 20 patients in group B, no local anesthetic was applied postharvest (Table 1).

Postoperative pain levels at the iliac crest were assessed using the visual analog scale (VAS) starting on postoperative day 1 until day 5 , at which point the patients were released from the hospital.

Routinely, patients with multilevel revision surgeries recovered at the intensive care unit (ICU).

During their stay at the ICU, morphine use by patientcontrolled analgesia (PCA), by piritramide administration, was calculated and recorded. Only patients who underwent an ICU stay of two days and who had no previous ICG harvesting procedures were included in our analysis. VAS data were collected from postoperative day $1-5$, and after six months patients were assessed during 

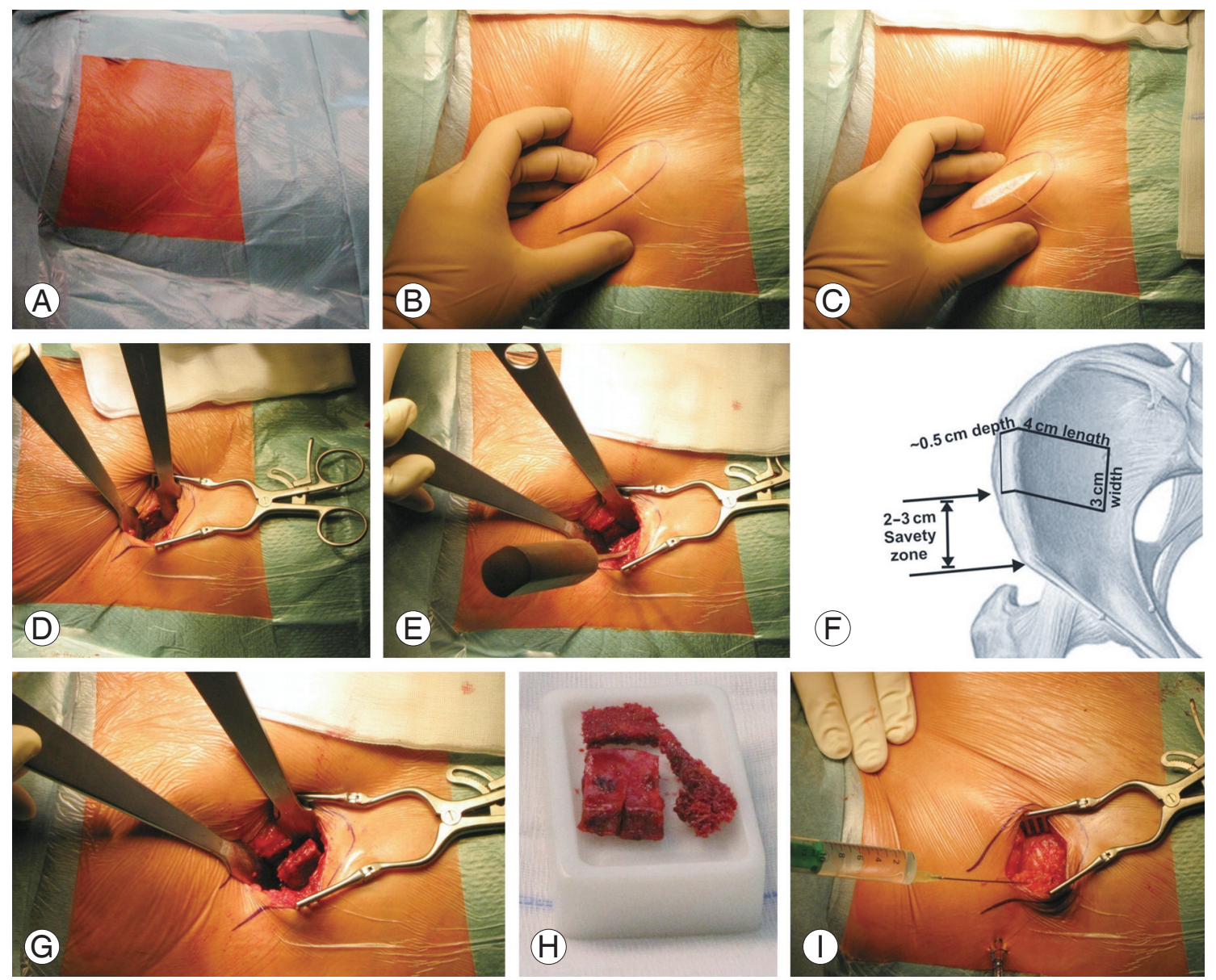

Fig. 1. (A-I) Surgical technique for iliac crest graft harvesting. In all cases, bicortical corticocancellous grafts were harvested from the inner iliac tablet approximately $2 \mathrm{~cm}$ proximal to the anterior superior iliac spine using an oscillating saw. Subsequently, bonewax was used to stop bleeding from the cancellous bone, and an absorbable sponge $(3 \times 5 \mathrm{~cm}$, Spongostan, Johnson\&Johnson, New Brunswick, USA) was inserted into the defect. After refixation of the abdominal fascia to the iliac crest, one surgeon administered $7-10 \mathrm{~mL}$ of ropivacaine (0.2\%) into the defect, the abdominal fascia and the iliac crest using a cannula (group A). The other patients constituted group $B$; this group underwent an identical procedure without the administration of ropivacaine.

Table 1. Patient characteristics and VAS scores

\begin{tabular}{|c|c|c|c|c|c|c|c|c|c|}
\hline \multirow{2}{*}{ Characteristic } & \multirow{2}{*}{$\begin{array}{c}\text { Age at } \\
\text { surgery } \\
\text { (yr) }\end{array}$} & \multirow{2}{*}{$\begin{array}{c}\text { No. of } \\
\text { previous } \\
\text { surgeries }\end{array}$} & \multirow{2}{*}{$\begin{array}{l}\text { No. of } \\
\text { levels } \\
\text { fused }\end{array}$} & \multirow{2}{*}{$\begin{array}{l}\text { Amount of } \\
\text { naropin } \\
(\mathrm{mL})\end{array}$} & \multicolumn{5}{|c|}{ VAS } \\
\hline & & & & & Day 1 & Day 2 & Day 3 & Day 4 & Day 5 \\
\hline Group A & $\begin{array}{c}56.0 \pm 7.6 \\
(43-68)\end{array}$ & $\begin{array}{c}0.6 \pm 0.7 \\
(0-2)\end{array}$ & $\begin{array}{c}3.6 \pm 0.7 \\
(2-5)\end{array}$ & $\begin{array}{l}8.4 \pm 1.5 \\
(4-10)\end{array}$ & $\begin{array}{c}1.4 \pm 2.3 \\
(0-7)\end{array}$ & $\begin{array}{c}2.5-1.7 \\
(0-5)\end{array}$ & $\begin{array}{l}3.5 \pm 2.2 \\
(0-8.5)\end{array}$ & $\begin{array}{c}3.0-2.1 \\
(0.5-10)\end{array}$ & $\begin{array}{l}3.1 \pm 2.7 \\
(0-10)\end{array}$ \\
\hline Group B & $\begin{array}{l}52.6 \pm 10.4 \\
(37-70)\end{array}$ & $\begin{array}{c}0.8 \pm 1.0 \\
(0-3)\end{array}$ & $\begin{array}{c}3.7 \pm 0.9 \\
(2-6)\end{array}$ & NA & $\begin{array}{c}3.9 \pm 2.4 \\
(0-8)\end{array}$ & $\begin{array}{c}4.6 \pm 2.1 \\
(1-8)\end{array}$ & $\begin{array}{c}4.2+2.1 \\
(0-8)\end{array}$ & $\begin{array}{c}3.9 \pm 2.0 \\
(0-7)\end{array}$ & $\begin{array}{c}3.5 \pm 2.3 \\
(0-8)\end{array}$ \\
\hline
\end{tabular}

Values are presented as mean \pm standard deviation (minimum-maximum) VAS, visual analog scale; NA, not applicable.

routine follow-up at the outpatient clinic.

Any complications at the ICG harvest site or related to the cervical spine surgery were recorded. Complications were stratified as minor or major. Major complications included non-union, neurological deterioration, need for revision, cerebrospinal fluid leakage, and fracture or infection at the harvest site. At six months postoperative, any problems at the ICG harvest site were noted, especially gait disturbances, wound healing problems and chronic pain. Biplanar radiographs of the cervical spine 
were assessed for fusion and construct stability. Patients who required surgical revision were noted.

\section{Statistics}

Means, standard deviations, medians, and 25\% and 75\% quartiles were used to compute the descriptive statistics. The Mann-Whitney $U$ test was used as a nonparametric test to compare the VAS scores of both groups at five days postoperatively. Spearman's rank correlation coefficients were computed together with corresponding tests. A $p$-value less than $5 \%$ was assumed to indicate statistical significance. All computations were performed using Statistica ver. 6.1 software (StatSoft, Tulsa, OK, USA).

\section{Results}

The average age of the patients in groups $A$ and $B$ was $56.0 \pm 7.6$ years and $52.6 \pm 10.4$ years, respectively. Group
A had an average of $0.6 \pm 0.7$ previous surgeries per patient, whereas group $B$ had an average of $0.8 \pm 1.0$ previous surgeries per patient. The average number of levels fused in group A was 3.6 \pm 0.7 , whereas the average number of levels fused in group B was 3.7 \pm 0.9 . We observed no significant differences in the baseline values between the two groups $(p>0.05)$.

In group $\mathrm{A}$, a mean ropivacaine volume of $8.4 \pm 1.5 \mathrm{~mL}$ was administered. On day 1 postoperative, the VAS at the ICG harvest site in patients in groups A and B were $1.4 \pm 2.3$ and $3.9 \pm 2.4$, respectively. On day 2 postoperative, the VAS was $2.5 \pm 1.7$ for group A and 4.6 \pm 2.1 for group B. On day 3 postoperative, the VAS was $3.5 \pm 2.2$ for group A and $4.2 \pm 2.1$ for group B. On day 4 postoperative, the VAS was 3.0 \pm 2.1 for group A and 3.9 \pm 2.0 for group B. On day 5 postoperative, the VAS was $3.1 \pm 2.7$ for group A and $3.5 \pm 2.3$ for group B. Values for pain at the ICG harvest site are illustrated in Fig. 2. In group A, three patients had a postoperative pain level $\geq 6$ points, whereas in group $B$,

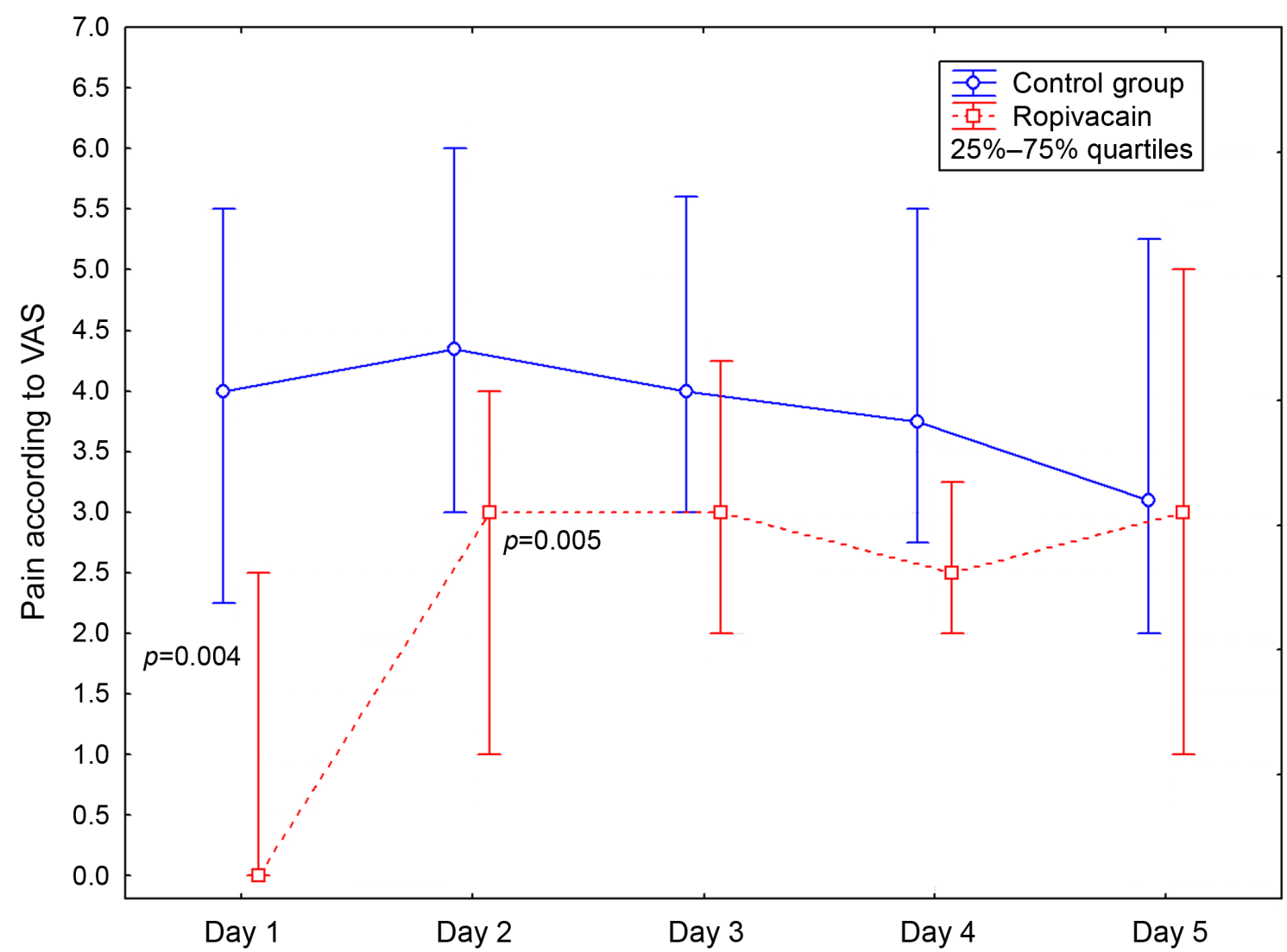

Fig. 2. Graph illustrating perioperative changes in pain at the iliac crest graft harvest site. The dashed red line indicates the ropivacaine group, and the blue line indicates the control group. Notably, patients in the ropivacaine group had less pain at all time points postoperative relative to the control group, and significant differences were observed for days 1 and 2 postoperative. VAS, visual analog scale. 
11 patients had a similar postoperative pain level $(p<0.05)$. In group $A$, of the patients who scored $\geq 6$ on the metric VAS scale, one patient was recorded on day 1 postoperative, zero patients were recorded on day 2 , two patients were recorded on day 3 , two patients were recorded on day 4 and three patients were recorded on day 5 . In group $B$, of the patients who scored $\geq 6$ on the metric VAS scale, five were recorded on day 1 , seven on day 2 , five on day 3 , five on day 4 , and four on day 5 .

Statistical analysis revealed a significant difference in VAS between group A and group B on day $1(p=0.004)$ and day $2(p=0.005)$. PCA morphine use was not significantly different $(p=0.58)$ between groups $A$ and $B$ and showed no correlation with the number of previous surgeries or the number of levels fused. In a statistical analysis of all patients, a weak correlation between PCA morphine use and patient age was observed $(p=0.034$, $r=0.3)$.

The amount of ropivacaine administered varied within a narrow range $(8.4 \pm 1.5 \mathrm{~mL})$ and showed no correlation with pain levels. Neither group A nor group B showed a correlation between age, number of previous surgeries or number of levels fused and VAS. The order of the cases within the prospective analysis also showed no correlation with the pain levels observed.

Six months after the surgery, no patient reported complaints regarding chronic pain at the ICG harvest site, and no gait disturbances or wound healing problems were detected. Two patients in each group presented with a postoperative C5-palsy that fully recovered prior to the 6-month follow-up. No anesthesiology-related complications were observed. Radiographic fusion was achieved in all patients. No complications that required surgical intervention were observed related to ICG harvesting.

\section{Discussion}

Complications related to anterior ICG harvesting have been reported previously and include pain, hematoma, infection, neuropraxia of the lateral femoral cutaneous, iliohypogastric and ilioinguinal nerves, fracture of the ilium, gait disturbances, peritoneal perforation, hernia and cosmetic deformity [8]. However, a standardized technique that implements various safety margins [9] can reduce these risks, as shown in the current study. Usually, postoperative pain is modest and can be ameliorated with standardized pain management protocols, includ- ing local ice packs in addition to intravenous and oral pain medications. We hypothesized that pain could be further reduced by an intraoperative administration of local anesthetics; however, the duration of the anesthetic effect was not clear. This prospective assessment showed a significant difference in pain levels for day 1 and day 2 after the surgery, and in total, only three patients in group A and four patients in group B reported pain levels $\geq 6$ by the fifth postoperative day, indicating that the pain at the ICG harvest site was generally moderate. This study was performed in a series of 40 patients who underwent multilevel anterior cervical spine surgery. Studies based on multilevel ACDFP are scarce; however, several authors have reported that fusion rates decrease as the number of levels included in the fusion mass increases [8]. In the current study, using biplanar radiographs, fusion and construct stability were maintained at six months in all patients. The results indicated that the use of autogenous bone in combination with a primary stable reconstruction technique corresponded to a high fusion rate. The incidence of fusion and the absence of non-union correlated with an improved clinical outcome in lumbar and in cervical fusion surgery $[10,11]$. Accordingly, the impact of various substitutes for autogenous bone on fusion rates remains a significant issue of debate until larger computed tomography scan endpoint-based studies determine the true fusion rates associated with these procedures.

In the current study, a moderate ICG-related morbidity persisted in the postoperative period but could be ameliorated with the local administration of an anesthetic. Morbidity was generally low and did not persist at the six-month follow-up, at which point no patients reported chronic pain. Comparable results were found by Skeppholm and Olerud [12], who reported that pain from anterior ICG harvesting did not seem to affect the quality of life at four weeks postoperative or at a final followup one year after surgery. Substitutes for autogenous bone avoid harvest site-related pain, but may decrease fusion rates. Furthermore they cause additional costs for the health care system adding to the concerns. A metaanalysis by Floyd and Ohnmeiss [8] showed a significant higher pseudarthrosis rate using allografts compared to autografts in one- and two-level ACDF.

In 251 patients with one-level ACDF, the pseudarthrosis rate using autografts was $6.0 \%$, whereas the corresponding pseudarthrosis rate using allografts was $14.7 \%$. In patients with two-level ACDF, $20 \%$ of patients using 
autografts and $45.8 \%$ using allografts presented with pseudarthrosis. This difference in pseudarthrosis is expected to be even more pronounced in multilevel surgeries. Bone morphogenetic proteins have shown considerable promise in lumbar spine surgery; however, routine usage in anterior cervical spine surgery is not performed due to reports of serious adverse events $[7,13]$.

Stress or avulsion fractures of the anterior ilium after the harvesting of bicortical or tricortical bone grafts have been reported [14-16] but did not occur in the current study. A morphological study of the iliac crest showed that bicortical bone blocks and cancellous bone may be best harvested from the superior and internal surfaces of the iliac tubercle [17]. Using an oscillating saw, less stress is imparted on the grafts, whereas the use of an osteotome might create cracks and predispose the iliac crest to stress or avulsion fractures [18]. The base bearing the anterior superior iliac spine must be large enough to resist the downward pull of the sartorius muscle. A distance of $30 \mathrm{~mm}$ between the anterior superior iliac spine and the harvest site preserves 2.4 times more strength in the iliac crest when compared with a distance of $15 \mathrm{~mm}$ [9]. The recommendations published in the aforementioned studies were incorporated in the surgical technique for ICG harvesting and might explain the low rate of complications and perioperative pain.

Anecdotal observations have shown that the proximity of lumbar fusion surgery to the posterior ICG harvest site may confound the effects of preventive local drug administration, because of patient difficulty in distinguishing hip and iliac crest pain from centralized spinal pain. These observations might explain the lack of effect of local anesthetics in lumbar spine surgery. Wai et al. [19] conducted a prospective study on 54 patients with intraoperative local administration of morphine or saline, and the authors found no additional benefits for the intraoperative injection of morphine into the ICG harvest site. However, only five patients in this study underwent cervical spine surgery with anterior ICG harvesting, whereas 49 patients were treated with lumbar fusions and posterior ICG harvesting. Sasso et al. [20] reported on 199 patients who underwent anterior ICG harvesting for anterior lumbar interbody fusion. No additional local measurements were taken in this study. Thirty-one percent of the patients reported persistent pain 24 months postoperative, and $16 \%$ of patients reported a fair or poor appearance of their graft site. No data on the early post- operative period were available. Cricchio and Lundgren [21] investigated the donor site morbidity of anterior ICG harvesting for atrophic edentulous maxilla. Eleven percent of the patients reported pain or discomfort two years after harvesting, and $4 \%$ of patients sustained a major complication (iliac wing fracture or neurological injury). In our study, we investigated the pain levels of 40 adult patients who underwent multilevel ACDFP. Contrary to Estebe et al. [22], who reported that pain from the ICG harvest site was significantly reduced for only 12 hours using $20 \mathrm{~mL}$ of ropivacaine in limb surgery, we found a significant difference in the VAS on day 1 and day 2 postoperative. The longer-lasting effect in our study sample could be due to a reservoir function of the absorbable sponge, which could have absorbed and gradually secreted ropivacaine to the surrounding tissue. Our results compare favorably to Dashow et al. [23], who compared a bupivacaine-soaked absorbable sponge combined with local bupivacaine administration to the administration of a single dose of bupivacaine in children who underwent alveolar cleft surgery. Patients who received an absorbable sponge had significantly lower mean pain scores and required less pain medication. Singh et al. [24] investigated the efficacy of a continuous infusion catheter administering saline or marcaine applied for 48 hours in 37 surgery patients (cervical surgery $n=9$ ). In this study, the VAS was significantly reduced in the patients who received marcaine at 24 and 48 hours postoperative, and PCA narcotic usage in patients who received marcaine was reduced by $50 \%$. Ouaki et al. [25] used a continuous infusion of $0.2 \%$ ropivacaine with disposable elastomeric pumps for 48 hours in 16 children that underwent maxillar alveolar graft surgery. Median pain scores were zero for all patients; however, one patient exhibited neuropathic pain symptoms at the three-month follow-up. Different results were found in a study performed by Morgan et al. [26], who used a continuous infusion of $0.5 \%$ bupivacaine. Sixty patients who underwent orthopedic trauma surgery reported no difference in the perceived pain levels. The patients in this study underwent anterior and posterior ICG harvesting, and pain was assessed until six weeks postoperative. Chiono et al. [27] used a preoperative ultrasound-guided transverse abdominal plane block in 33 patients. VAS was evaluated for 48 hours, and the average score was zero (range, 0-7). However, at the 18-month follow-up, $20 \%$ of patients reported chronic pain (VAS $2-4$ ), and $26 \%$ of patients complained about numbness. 
No such complications were encountered in our patient sample. Barkhuysen et al. [28] administered a single dose of bupivacaine or no treatment to patients who underwent ICG harvesting for cranio-maxillofacial surgery. The authors reported no differences concerning postoperative pain and gait disturbance; however, only 98 questionnaires out of 200 consecutive patients were eligible, which may have confounded the results.

\section{Conclusions}

In the current study of 40 patients who underwent multilevel ACDFP with an average of 3.7 levels, morbidity at the donor site was generally moderate and, based on the high fusion rate, deemed justifiable. Pain during the first two postoperative days can be further diminished with a single intraoperative injection of local anesthetics without raising the rate of complications. A mean pain level of 4.0 in the patients who did not receive ropivacaine was reduced to 2.7 in the patients who received ropivacaine.

\section{References}

1. Koller H, Hempfing A, Ferraris L, Maier O, Hitzl W, Metz-Stavenhagen P. 4- and 5-level anterior fusions of the cervical spine: review of literature and clinical results. Eur Spine J 2007;16:2055-71.

2. Brazenor GA. Comparison of multisegment anterior cervical fixation using bone strut graft versus a titanium rod and buttress prosthesis: analysis of outcome with long-term follow-up and interview by independent physician. Spine (Phila Pa 1976) 2007;32:63-71.

3. Chau AM, Mobbs RJ. Bone graft substitutes in anterior cervical discectomy and fusion. Eur Spine J 2009;18:449-64.

4. Fineberg SJ, Ahmadinia K, Oglesby M, Patel AA, Singh K. Hospital outcomes and complications of anterior and posterior cervical fusion with bone morphogenetic protein. Spine (Phila Pa 1976) 2013;38: 1304-9.

5. Lu DC, Tumialan LM, Chou D. Multilevel anterior cervical discectomy and fusion with and without rhBMP-2: a comparison of dysphagia rates and outcomes in 150 patients. J Neurosurg Spine 2013;18:439.

6. Perri B, Cooper M, Lauryssen C, Anand N. Adverse swelling associated with use of rh-BMP-2 in anterior cervical discectomy and fusion: a case study. Spine J 2007;7:235-9.

7. Fehlings MG, Arvin B. Surgical management of cervical degenerative disease: the evidence related to indications, impact, and outcome. J Neurosurg Spine 2009;11:97-100.

8. Floyd T, Ohnmeiss D. A meta-analysis of autograft versus allograft in anterior cervical fusion. Eur Spine J 2000;9:398-403.

9. Hu R, Hearn T, Yang J. Bone graft harvest site as a determinant of iliac crest strength. Clin Orthop Relat Res 1995;(310):252-6.

10. Kuhns CA, Geck MJ, Wang JC, Delamarter RB. An outcomes analysis of the treatment of cervical pseudarthrosis with posterior fusion. Spine (Phila Pa 1976) 2005;30:2424-9.

11. Kaiser MG, Mummaneni PV, Matz PG, et al. Management of anterior cervical pseudarthrosis. J Neurosurg Spine 2009;11:228-37.

12. Skeppholm M, Olerud C. Pain from donor site after anterior cervical fusion with bone graft: a prospective randomized study with 12 months of follow-up. Eur Spine J 2013;22:142-7.

13. Carragee EJ, Hurwitz EL, Weiner BK. A critical review of recombinant human bone morphogenetic protein-2 trials in spinal surgery: emerging safety concerns and lessons learned. Spine J 2011;11:47191.

14. Covani U, Ricci M, Santini S, Mangano F, Barone A. Fracture of anterior iliac crest following bone graft harvest in an anorexic patient: case report and review of the literature. J Oral Implantol 2013;39:103-9.

15. $\mathrm{Hu} \mathrm{RW}$, Bohlman $\mathrm{HH}$. Fracture at the iliac bone graft harvest site following fusion of the spine. Clin Orthop Relat Res 1994;(309):208-13.

16. Guha SC, Poole MD. Stress fracture of the iliac bone with subfascial femoral neuropathy: unusual complications at a bone graft donor site: case report. Br J Plast Surg 1983;36:305-6.

17. Ebraheim NA, Yang H, Lu J, Biyani A, Yeasting RA. Anterior iliac crest bone graft. Anatomic considerations. Spine (Phila Pa 1976) 1997;22:847-9.

18. Jones AA, Dougherty PJ, Sharkey NA, Benson DR. Iliac crest bone graft. Osteotome versus saw. Spine (Phila Pa 1976) 1993;18:2048-52.

19. Wai EK, Sathiaseelan S, O’Neil J, Simchison BL. Local administration of morphine for analgesia after 
autogenous anterior or posterior iliac crest bone graft harvest for spinal fusion: a prospective, randomized, double-blind, placebo-controlled study. Anesth Analg 2010;110:928-33.

20. Sasso RC, LeHuec JC, Shaffrey C; Spine Interbody Research Group. Iliac crest bone graft donor site pain after anterior lumbar interbody fusion: a prospective patient satisfaction outcome assessment. J Spinal Disord Tech 2005;18 Suppl:S77-81.

21. Cricchio G, Lundgren S. Donor site morbidity in two different approaches to anterior iliac crest bone harvesting. Clin Implant Dent Relat Res 2003;5:161-9.

22. Estebe JP, Le Corre P, Le Naoures A, et al. Local anaesthetic use for the iliac crest-donor site: pharmacokinetic and pharmacodynamic evaluations. Acta Anaesthesiol Belg 2009;60:39-45.

23. Dashow JE, Lewis CW, Hopper RA, Gruss JS, Egbert MA. Bupivacaine administration and postoperative pain following anterior iliac crest bone graft for alveolar cleft repair. Cleft Palate Craniofac J 2009;46:173-8.

24. Singh K, Phillips FM, Kuo E, Campbell M. A prospective, randomized, double-blind study of the effi- cacy of postoperative continuous local anesthetic infusion at the iliac crest bone graft site after posterior spinal arthrodesis: a minimum of 4-year follow-up. Spine (Phila Pa 1976) 2007;32:2790-6.

25. Ouaki J, Dadure C, Bringuier S, et al. Continuous infusion of ropivacaine: an optimal postoperative analgesia regimen for iliac crest bone graft in children. Paediatr Anaesth 2009;19:887-91.

26. Morgan SJ, Jeray KJ, Saliman LH, et al. Continuous infusion of local anesthetic at iliac crest bone-graft sites for postoperative pain relief. A randomized, double-blind study. J Bone Joint Surg Am 2006;88: 2606-12.

27. Chiono J, Bernard N, Bringuier S, et al. The ultrasound-guided transversus abdominis plane block for anterior iliac crest bone graft postoperative pain relief: a prospective descriptive study. Reg Anesth Pain Med 2010;35:520-4.

28. Barkhuysen R, Meijer GJ, Soehardi A, et al. The effect of a single dose of bupivacaine on donor site pain after anterior iliac crest bone harvesting. Int J Oral Maxillofac Surg 2010;39:260-5. 